\title{
Estudo de demanda em unidades de Saúde da Família em cidade de porte médio do estado do Rio Grande do Sul, Brasil
}

\author{
Elaine Tomasi, Alexsandro Behrens Zibel, Valquiria Porto Garcez, Rogério da Silva Linhares, \\ Ângela Moreira Vitória.
}

\section{RESUMO}

Objetivo: Descrever o perfil da demanda de Unidades Básicas de Saúde (UBS).

Métodos: Foram registrados todos os atendimentos em cinco unidades de saúde durante cinco semanas, incluindo sexo, idade e motivos de atendimentos.

Resultados: Foram atendidas 7.754 pessoas, o que representou 310 por dia e estimativa anual de 80.639 . Foram realizados 4.817 atendimentos médicos, com média diária de 193 e estimativa anual de 50.927. A maioria (68\%) era constituída por mulheres e houve predomínio das faixas etárias de 50 a 69 anos (30\%) e 18 a 29 anos (16\%). Os tipos de atendimento médico mais frequentes foram: consulta clínica (53\%), renovação de receita (22\%) e pronto atendimento (4\%).

Conclusões: As UBS estudadas são similares às demais de município de porte médio, em termos de modelo de atenção e de cobertura populacional, o que torna os resultados com potencial de extrapolação para o município como um todo e para outros de porte similar.

Palavras-chave: Necessidades e Demandas de Serviços de Saúde, atenção primária em saúde; Saúde da Família.

\section{ABSTRACT}

Aim: To describe the profile of the demand for Basic Health Units (UBS).

Methods: All visits made by five health units during five weeks were recorded, including sex, age and reasons for visits.

Results: 7,754 people were included, representing 310 per day and an annual estimate of 80,639. 4,817 medical visits were performed, with a daily average of 193 and an annual estimate of 50,927 . The majority $(68 \%)$ were women and there was a predominance of the age groups from 50 to 69 years old (30\%) and 18 to 29 years old $(16 \%)$. The most frequent types of medical care were: clinical consultation $(53 \%)$, prescription renewal (22\%) and emergency care (4\%).

Conclusions: The UBS studied are similar to the others in a mediumsized municipality, in terms of the care model and population coverage, which makes the results with extrapolation potential for the municipality as a whole and for others of similar size.

Keywords: Health Services Needs and Demand, Primary Health Care, Family Health.
Revista da Rede APS 2021

Publicada em: 01/04/2021

DOI:10.14295/aps.v3i1.56

Elaine Tomasi

(Departamento de Medicina Social, Universidade Federal de Pelotas, Pelotas, RS, Brasil)

Alexsandro Behrens Zibel (Faculdade de Medicina, Universidade Federal de Pelotas, Pelotas, RS, Brasil)

Valquiria Porto Garcez (Faculdade de Medicina, Universidade Federal de Pelotas, Pelotas, RS, Brasil)

Rogério da Silva Linhares (Departamento de Medicina Social, Universidade Federal de Pelotas, Pelotas, RS, Brasil)

Ângela Moreira Vitória (Departamento de Medicina Social, Universidade Federal de Pelotas, Pelotas, RS, Brasil)

Correspondência para:

Elaine Tomasi tomasiet@gmail.com 


\section{INTRODUÇÃO}

A Declaração de Alma Ata, em 1978, oficializou a Atenção Primária a Saúde (APS) como estratégia prioritária para ampliar o acesso à saúde (ANDRADE et al., 2015; SANTOS; RIBEIRO, 2015; STARFIELD, 2002). Neste contexto, a $8^{a}$ Conferência Nacional de Saúde em 1986 no Brasil lançou as bases do Sistema Único de Saúde (SUS) e deliberou que a saúde é direito de todo cidadão, com foco na APS, migrando de um modelo assistencial tradicional centrado na doença e no cuidado médico individualizado para um modelo que prioriza a atenção primária e seus princípios e diretrizes (PAIM et al., 2011). A APS, aqui utilizada como sinônimo de Atenção Básica ( $A B)$, caracteriza-se por um conjunto de ações individuais e coletivas sobre os problemas mais prevalentes na comunidade. Objetiva a prevenção e o controle de enfermidades, bem como a promoção, diagnóstico, tratamento e reabilitação dos usuários. (BRASIL, 1998; ESPERANÇA; CAVALCANTE; MARCOLINO, 2006; GUIBU et al., 2017; STARFIELD, 2002). Suas ações são desenvolvidas de forma descentralizada por meio de práticas de gestão e cuidado voltadas às populações adscritas, fazendo uso de tecnologias em saúde compatíveis com a realidade da comunidade, suas demandas e necessidades (BRASIL, 2012).

Ainda que muito se discuta sobre prevenção, promoção e cuidado na organização dos serviços, ainda são escassos estudos que tratem da operacionalização das rotinas assistenciais na Estratégia Saúde da Família (ESF) (NORMAN; TESSER, 2015). Este elo entre a discussão e a operacionalização se dá pelo planejamento ao se considerar as necessidades de reorganização dos serviços de acordo com demandas geradas e tendo em vista o perfil dos usuários, tratandoos em sua singularidade, complexidade e integralidade (BRASIL, 2012).

O objetivo deste artigo é descrever o perfil dos usuários atendidos em Unidades Básicas de Saúde (UBS) da Universidade Federal de Pelotas (UFPel), os tipos e os motivos de atendimento, a fim de reunir subsídios para ações de planejamento e avaliação da atenção à saúde em processo contínuo de melhoria da qualidade. Os resultados podem apoiar gestores a redirecionar recursos humanos, equipamentos e suprimentos mais adequados às necessidades dos serviços de saúde, incluindo a qualificação de suas equipes.

\section{MÉTODOS}

O estudo teve delineamento transversal com todos os atendimentos realizados por todos os membros das equipes das cinco unidades de saúde sob responsabilidade da Universidade Federal de Pelotas - Areal Leste, Centro Social Urbano do Areal, Obelisco, Vila Municipal e Campus Capão do Leão - durante cinco semanas entre novembro e dezembro de 2018, independentemente de residência na área de abrangência da Unidade.

Após estudo piloto e por meio de formulários impressos, foram coletados dados sobre os usuários e os atendimentos, cujos motivos foram classificados de acordo com a Classificação Internacional de Doenças - 10ạ. Revisão - CID-10. Sexo e idade foram registrados para os usuários na recepção e para os atendidos em consultas médicas e de enfermagem. Além destas consultas, foram considerados outros tipos de atendimento médico - urgência, visita domiciliar, renovação de receita e procedimentos ambulatoriais - e de enfermagem - prática assistencial, prática administrativa e atividades de promoção e de educação em saúde. Todos os atendimentos de nutricionistas, assistentes sociais, odontólogos e psicólogos também foram caracterizados para o período. As UBS recebem alunos para estágios curriculares de cursos de graduação e residentes de Medicina de Família e Comunidade e de Psiquiatria. Todos os profissionais foram capacitados para o completo preenchimento dos campos nos formulários.

Ao final de cada semana do estudo, os formulários eram recolhidos nas UBS, quando também se iniciou a montagem dos bancos de dados para processamento em pacote estatístico, mas, por tratar-se de estudo 
descritivo, não foram empregados testes de hipóteses.

As informações não identificaram as pessoas, o que fez com que o trabalho respeite a privacidade e garanta o caráter confidencial das informações. Assim, foi solicitado ao Comitê de Ética dispensa da utilização do Termo de Consentimento Livre e Esclarecido, o que foi atendido, conforme parecer 94230618.0.0000.5317.

\section{RESULTADOS}

No período, foram atendidas 7.754 pessoas nas cinco UBS, sendo 310 pessoas por dia com estimativa anual de 80.639 usuários. Do total de pessoas atendidas, a maioria (68\%) era constituída por mulheres e houve predomínio das faixas etárias de 50 a 69 anos (30\%) e 18 a 29 anos (16\%) (Figura 1). Os grupos com maior afluência às UBS foram mulheres de 18 a 29 anos $(18,1 \%)$ e homens de 60 a 69 anos (17,5\%). Nas faixas etárias de até 17 anos houve predomínio de homens e na faixa de 18 a 59 anos, o predomínio foi de mulheres.

Figura 1 - Pessoas atendidas conforme o sexo e o grupo etário. Unidades Básicas de Saúde, UFPEL, Pelotas, RS, 2018 ( $n=7.754)$.

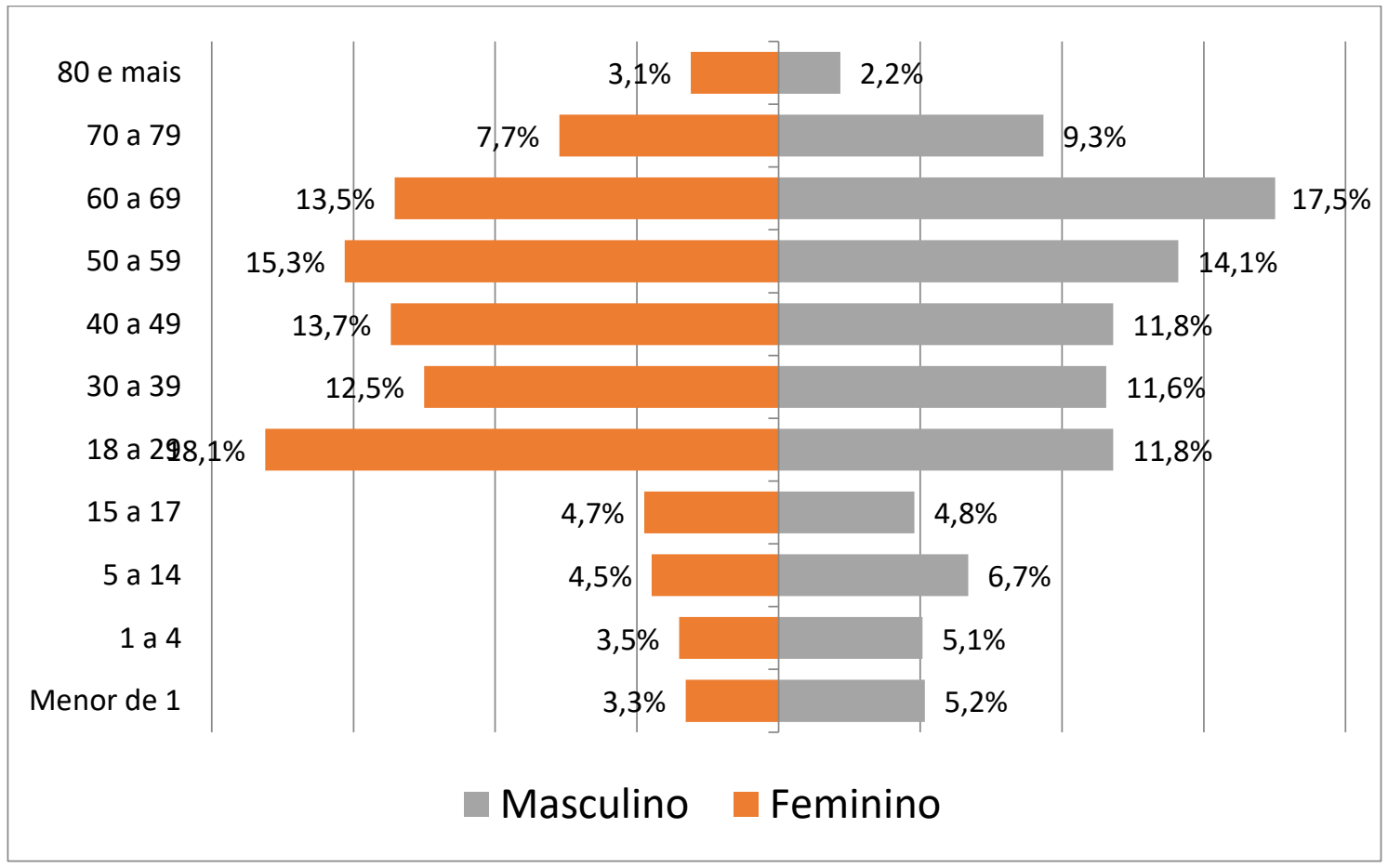

Fonte: Dados do inquérito, elaboração dos autores

Para o conjunto destes usuários foram realizados 4.817 atendimentos médicos, com média diária de 193 e estimativa anual de 50.927 atendimentos. Mulheres entre 18 e 29 anos (20\%) e homens entre 60 e 69 anos (20\%) foram os que mais receberam atendimentos médicos. Meninos com idade inferior a 14 anos representaram $16 \%$ dos atendimentos enquanto meninas desta faixa etária representaram 9\%. Entre 15 e 59 anos, as mulheres foram a maioria da demanda atendida (64\%), mas a partir dos 60 anos foram os homens (34\%) os indivíduos mais atendidos nas unidades de saúde, frente a $26 \%$ das mulheres. Os tipos de atendimento médico mais frequentes foram: consulta clínica (53\%), 
renovação de receita (22\%) e pronto atendimento (4\%). (Figura 2)

De acordo a CID-10, os principais grupos foram: exames e investigações - Capítulo XXI (21\%), queixas do aparelho circulatório - Capítulo IX (15\%), transtornos mentais - Capítulo V (13\%) e doenças endocrinológicas, metabólicas e nutricionais - Capítulo IV (8\%). Analisado o capítulo XXI, vimos que "exame em pessoas sem queixas prévias" correspondeu a $27 \%$ dos atendimentos, seguido de "supervisão de gravidez normal" (22\%), "prescrição de repetição" (15\%) e "rastreamento de câncer de colo uterino" (14\%).

Dentro do capítulo do aparelho circulatório, predominou a hipertensão arterial sistêmica (78\%); a cardiopatia isquêmica e a insuficiência cardíaca somaram 9\%. Já entre os transtornos mentais prevaleceram os episódios de depressão (58\%) e os de ansiedade (17\%). No capítulo das doenças endócrino, metabólica e nutricionais, as mais frequentes foram o diabetes (52\%), o hipotireoidismo e a obesidade, ambos com $12 \%$ cada uma.

Foram realizados 4.584 atendimentos de enfermagem, dos quais $18 \%$ foram monitorização de sinais vitais, $11 \%$ administração de medicamentos, $11 \%$ dispensação de medicamentos e $10 \%$ aplicação de vacinas, todos no grupo de procedimentos de prática assistencial (89\%). A prática administrativa foi responsável por $7 \%$ e as atividades de promoção e educação em saúde por $4 \%$ de todos os atendimentos de enfermagem.

Figura 2 - Proporção dos atendimentos médicos $(n=4.817)$ e de enfermagem $(n=4.585)$ realizados em cinco semanas nas Unidades Básicas de Saúde, UFPEL, Pelotas, RS, 2018.

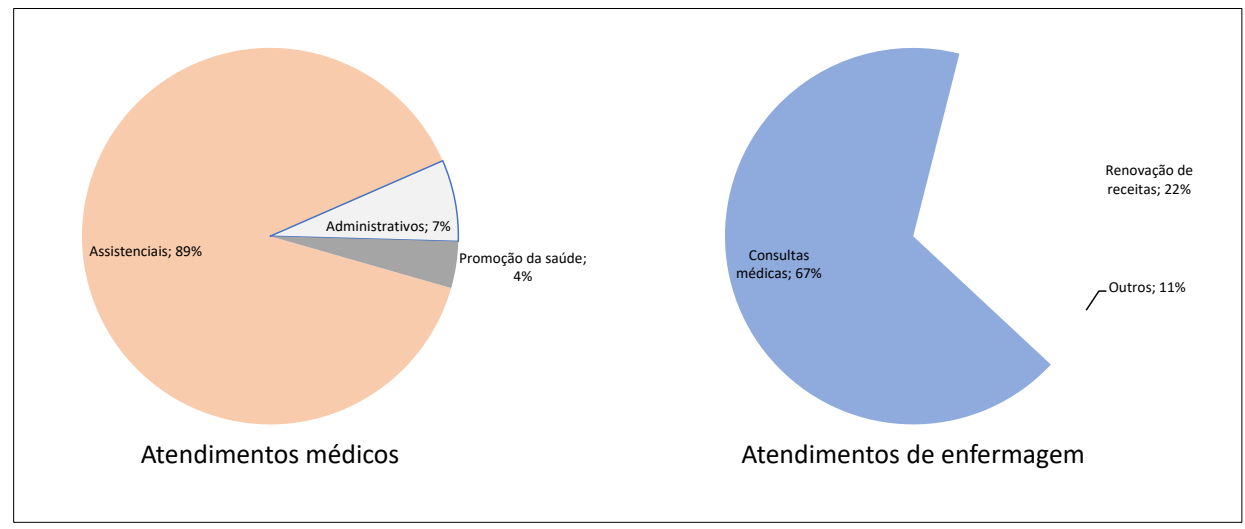

Fonte: Dados do inquérito, elaboração dos autores

Nutricionistas realizaram 277 atendimentos, com predomínio de consultas dietoterápicas (64\%) e educação em saúde (19\%); assistentes sociais foram responsáveis por 362 atendimentos, com destaque para as interconsultas (32\%) e entrevistas por problemas junto ao sistema de saúde (16\%); odontólogos fizeram 339 atendimentos, sendo $34 \%$ restaurações, $18 \%$ suturas e $16 \%$ limpeza ou raspagem; os psicólogos realizaram 56 atendimentos, com predomínio de atendimentos em grupo (32\%), discussão de casos da equipe (25\%) e atendimento individual (20\%)(Figura3).

Considerando uma estimativa anual de 50.927 atendimentos médicos para uma população das áreas de abrangência das UBS de 26.300 habitantes, obteve-se o indicador de 1,9 atendimentos/habitante/ano. Entretanto, ao considerar apenas as 33.331 consultas médicas estimadas no período, o indicador de consultas/habitante/ano ficou em 1,3. 
Figura 3 - Distribuição dos atendimentos de Nutrição, Serviço Social, Odontologia e Psicologia de acordo com a tipologia. UBS da UFPEL, Pelotas, RS, 2018.
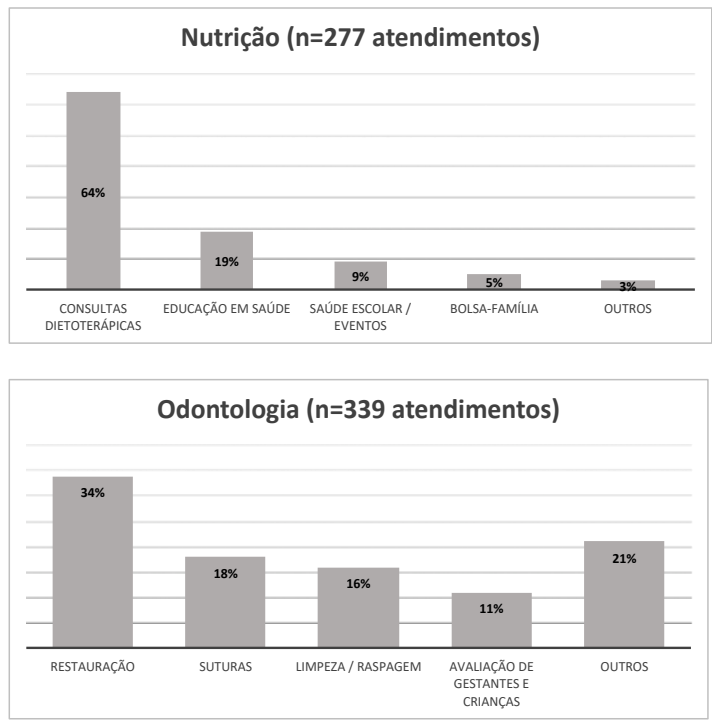

Fonte: Dados do inquérito, elaboração dos autores

\section{DISCUSSÃO}

Os estudos de demanda realizados em estabelecimentos de atenção primária são úteis para caracterizar o perfil dos usuários e avaliar a qualidade do cuidado dispensado. Nesta amostra, captou-se um volume de usuários e de atendimentos de cerca de $10 \%$ do total anual, supondo homogeneidade sazonal, o que pode ter influenciado no padrão dos motivos de consulta e das características dos usuários. $\mathrm{O}$ estudo ocorreu na primavera, mas o inverno no sul do Brasil faz com que as doenças respiratórias sejam mais prevalentes, atingindo mais crianças e idosos, o que poderia potencialmente modificar os resultados.

A média de consultas médicas por habitante por ano, sugerida nos parâmetros assistenciais do Sistema Único de Saúde (SUS), é de duas a três consultas por habitante/ano (BRASIL, 2015), bem maior do que o registrado em nosso estudo. O número médio consultas é um indicador importante para avaliar a qualidade da oferta de atenção à saúde na sua equipe. Ele pode ser influenciado por fatores socioeconômicos, epidemiológicos e demográficos, tais como nível de renda, perfil de morbidade, composição etária; infraestrutura

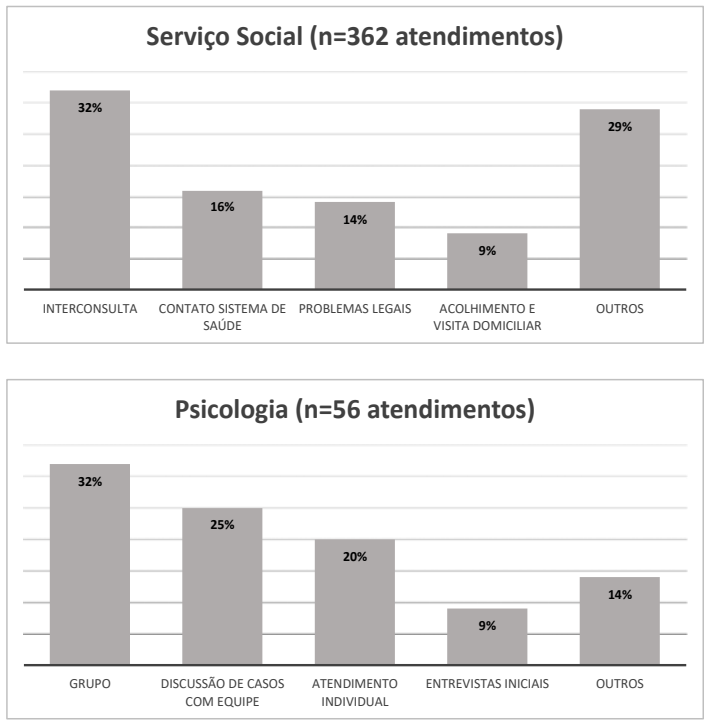

de serviços, com relação à disponibilidade de recursos humanos, materiais, tecnológicos, financeiros, políticas públicas assistenciais e preventivas, tais como critérios técnicoadministrativos de pagamento adotados no âmbito do SUS em cada município, bem como as desigualdades existentes na cobertura assistencial no município. Em 10 centros urbanos investigados por ocasião da implantação da Estratégia de Saúde da Família, os autores observaram grande variabilidade no número de consultas médicas, de 1,7 a 4,5 consultas/habitantes/ano. Em Goiânia, a média de consultas foi inferior à sugerida nos parâmetros assistenciais do SUS e superior em Vitória e Aracaju (ESCOREL et al., 2005).

Apesar da amostra de serviços ter sido intencional, as UBS estudadas são similares às demais deste município de porte médio, em termos de modelo de atenção e de cobertura populacional. Este fato permite extrapolar os achados deste estudo para o município como um todo e para outros municípios de porte similar.

Os resultados indicaram maior frequência de usuários do sexo feminino, similar ao perfil dos usuários referidos por outros autores (GUIBU et 
al., 2017; GUSSO, 2009; LANDSBERG et al., 2012; MAGNAGO et al., 2009; MIRANDA; SANTOS; CHAZAN, 2016; PIMENTEL et al., 2012; SANTOS; RIBEIRO, 2015; TOMASI et al., 2011; TORRES et al., 2015). Mulheres apresentam mais demanda por serviços de saúde para atender necessidades a seus ciclos reprodutivos e podem ter mais facilidade de acesso em função de seu trabalho, predominantemente domiciliar e do cuidado com os filhos, além de apresentarem maior preocupação com ações preventivas em saúde.

Em relação à idade, mais de $50 \%$ das pessoas atendidas tinha mais de 40 anos. A média de idade foi de 43 anos ( \pm 22 anos), sendo similar ao relato de TORRES et al., (2015), que foi de 46 anos ( \pm 16 anos). Estes achados estão condizentes com o padrão esperado em função da transição demográfica pela qual o país vem passando, com o consequente aumento da demanda por serviços de saúde entre pessoas com mais idade (BRASIL, 2015, 2019a). Além do declínio biológico das pessoas, aumenta a carga de exposição a fatores de risco - como o tabagismo, a inatividade física e a obesidade (BRASIL, 2015, 2019a), resultando em maior suscetibilidade a agravos crônicos. Além disso, ações de rastreio como a mamografia são preconizadas, no Brasil, para mulheres com mais 50 anos.

Também o conjunto de serviços ofertados pela rede básica pode conformar o padrão da demanda, com predomínio de mulheres jovens em busca de atenção à saúde reprodutiva (GUSSO, 2009; LANDSBERG et al., 2012; TOMASI et al., 2011). Entre os homens, prevalecem os atendimentos em idades mais avançadas, refletindo da mesma forma a escassez de ações direcionadas a esta parcela da população (GUIBU et al., 2017; LANDSBERG et al., 2012).

Para classificação dos motivos de atendimento médico, utilizou-se a CID-10 (ORGANIZAÇÃO MUNDIAL DE SAÚDE, 2008a), uma vez que as equipes das unidades de saúde investigadas também a utilizavam para registro rotineiro dos atendimentos. Em relação ao principal grupo de motivos - exames e investigações - os resultados não diferiram de outros estudos que utilizaram a Classificação Internacional da Atenção Primária (CIAP-2) (LANDSBERG et al., 2012; SANTOS; RIBEIRO, 2015; TORRES et al., 2015). Já em relação aos demais grupos de motivos, houve divergência, com os motivos de ordem respiratória ( $9 \%$ e $11 \%$ ) e digestiva ( $8 \%$ e $11 \%$ ) (LANDSBERG et al., 2012; SANTOS; RIBEIRO, 2015) ocupando o segundo e terceiro lugar, respectivamente. Em outros estudos predominaram os problemas musculoesqueléticos, circulatórios (GUSSO, 2009) ou neurológicos (TORRES et al., 2015), provavelmente devido às singularidades regionais ou de acordo com a sazonalidade esperada para determinadas afecções. Também é provável que, em nosso estudo, algumas queixas circulatórias possam ter sido classificadas como "exames e investigações". Este padrão sugere que predomina nas unidades o atendimento à demanda espontânea, sendo de volume maior do que de ações programáticas direcionadas a grupos específicos, como gestantes, puérperas, crianças e idosos.

Chamou atenção a elevada proporção dos atendimentos médicos dedicados à renovação de receitas. $\mathrm{O}$ adequado exercício dos atributos da APS como acesso de primeiro contato e longitudinalidade do cuidado aponta para equipes que conheçam a sua comunidade e ofereçam acesso facilitado, para inclusive, manter tratamentos já avaliados, prescritos e em condição adequada de uso, respeitando autonomia do paciente no manejo dos seus cuidados de saúde. As UBS em questão normatizaram que, a cada três meses, o paciente precisa ter consulta médica para renovar suas receitas, mas nos intervalos, poderá receber as receitas apenas solicitando na recepção da UBS. O estímulo à autonomia do paciente nos induz a confiar na sua avaliação e não exigir avaliação médica para manter o tratamento. Pode ser que o paciente apenas solicite a renovação da receita e não uma nova avaliação por causa da dificuldade de acesso e não pelas boas condições de saúde. Além de representar uma carga importante de trabalho na rotina das equipes, a renovação de medicamentos de uso contínuo pode implicar em riscos e potenciais danos à saúde, quando 
praticada sem protocolos e sem a avaliação clínica pertinente. Apesar de ser uma prática habitual em diversos serviços de atenção primária à saúde, a alta demanda por renovações de receita pode inviabilizar a abordagem integral do quadro clínico do usuário, sobretudo em situações nas quais não se conta com um prontuário adequado e tempo hábil para entrevista e avaliação do paciente. É frequente que muitos usuários não agendem consultas, não sejam atendidos presencialmente pelos médicos e tenham suas receitas renovadas, transcritas e medicações mantidas sem avaliação da adesão e de possíveis efeitos colaterais e interações medicamentosas. (SAASTAMOINEN; ENLUND; KLAUKKA, 2008) No Brasil, além de barreiras explicitadas no Código de Ética Médica - artigos 37 e 80 - (BRASIL, 2019b), ocupa-se parte da agenda dos profissionais com uma atividade que não gera benefício direto à população, podendo transmitir a sensação de que a população está sendo bem assistida apenas pelo fato de as pessoas conseguirem receber medicamentos, ainda que sem uma avaliação periódica.

Por outro lado, a prevalência de problemas de saúde mental é alta, chegando a aproximadamente $15 \%$ da população adulta em um estudo publicado pela OMS intitulado "Integração da Saúde Mental nos cuidados primários". (ORGANIZAÇÃO MUNDIAL DE SAÚDE, 2008b). Da mesma forma, as prevalências de hipertensão e diabetes chegam a $32 \%$ e $7,5 \%$ da população adulta, respectivamente (FLOR; CAMPOS, 2017; MALTA et al., 2018). Este fato, por si só, gera alta demanda de necessidade de acesso a medicação. Não temos conhecimento da proporção destes pacientes que estão com suas patologias compensadas e já adquiriram autonomia no seu cuidado. Aos pacientes autônomos e compensados, o fornecimento das receitas é qualidade do sistema. A questão que se coloca é de como é feita a avaliação da autonomia do paciente e da situação da patologia em questão.

Os profissionais de enfermagem são elementoschave na Estratégia de Saúde da Família, tendo sob sua carga ações de acolhimento, assistenciais (vacinação, realização de curativos, verificação de sinais vitais, testes rápidos) e atividades administrativas (organização do espaço, reuniões e capacitações de equipe). Destacou-se neste estudo a baixa proporção de atividades de promoção da saúde, sendo o tempo destes profissionais em sua maior parte dedicado a ações assistenciais e administrativas. Parece que, mesmo em um modelo de atenção voltado para a família e a comunidade, a demanda cotidiana ainda reflete a predominância do modelo biomédico, em que o cuidado se dá mais por meio de medidas e de procedimentos técnicos, de diagnósticos terapêuticos, comuns, em geral, em ambiente hospitalar (BARBIANI; NORA; SCHAEFER, 2016; MATUMOTO et al., 2012).

Apesar de que nas UBS estudadas já estivesse implantado o Prontuário Eletrônico do Cidadão - e-SUS, não foi possível utilizar as informações disponíveis em seus relatórios, tendo-se optado pela coleta de dados por meio de formulários em papel. Os relatórios emitidos pelo e-SUS não permitem cruzamento de dados, fornecendo apenas frequências simples das variáveis, apresentando também incompletude dos campos. Uma vez que os formulários foram preenchidos pelos próprios profissionais que realizavam os atendimentos, pode ter ocorrido problemas para a padronização dos dados, em que pese a capacitação realizada. Além disso, o estudo foi realizado em cinco UBS e não foi possível comparar o perfil demográfico de cada uma delas com a população em geral de forma a garantir a extrapolação dos resultados da pesquisa, o que só pode ser estimado.

Acredita-se que os resultados possam subsidiar gestores e equipes de Saúde da Família em seu planejamento de necessidades de recursos humanos e materiais, de modo a propiciar uma atenção à saúde mais efetiva à população sob sua adscrição. Também se sugere que fontes de dados como o e-SUS permitam, o mais breve possível, fornecer dados de forma sistematizada para apoiar a organização e o planejamento dos serviços de saúde para dar suporte às equipes de saúde da família. 


\section{REFERÊNCIAS}

ANDRADE, Mônica Viegas et al. A equidade na cobertura da Estratégia Saúde da Família em Minas Gerais, Brasil. Cadernos de Saúde Pública, [s. I.], v. 31, n. 6, p. 1175-1187, 2015. Disponível em: <https://doi.org/10.1590/0102-311X00130414>

BARBIANI, Rosangela; NORA, Carlise Rigon Dalla; SCHAEFER, Rafaela. Práticas do enfermeiro no contexto da atenção básica: Scoping review. Revista Latino-Americana de Enfermagem, [s. I.], v. 24, 2016. Disponível em: <https://doi.org/10.1590/1518-8345.0880.2721>

BRASIL. Portaria n.o 3.925, de 13 de novembro de 1998. Aprova o Manual para a Organização da Atenção Básica no Sistema Único de Saúde,. 1998. Disponível em: <http://bvsms.saude.gov.br/bvs/saudelegis/gm/1998/prt3925_13_11_1998_rep.html>.

Acesso em: 19 maio. 2018.

BRASIL. Política Nacional de Atençao Básica. [s.l: s.n.]. v. I Disponível em: <http://189.28.128.100/dab/docs/publicacoes/geral/pnab.pdf>

BRASIL. Pesquisa nacional de saúde : 2013 : ciclos de vida : Brasil e grandes regiõesRevista de Ciência Elementar. [s.I: s.n.]. Disponível em: <https://biblioteca.ibge.gov.br/visualizacao/livros/liv94522.pdf>.

BRASIL. Vigitel Brasil 2018: vigilância de fatores de risco e proteção para doenças crônicas por inquerito telefônico: estimativas sobre frequência e distribuição sociodemográfica de fatores de proteção para doenças crônicas nas capitais dos 26 estados brasileiro. [s.l: s.n.]. Disponível em: <http://bvsms.saude.gov.br/bvs/publicacoes/vigitel_brasil_2011_fatores_risco_doencas_cronicas.pdf>

BRASIL. Conselho Federal de Medicina. Código de Ética Médica: Resolução CFM no 2.217, de 27 de setembro de 2018 , modificada pelas Resoluções CFM no 2.222/2018 e 2.226/2019. Brasília. Disponível em: <https://portal.cfm.org.br/images/PDF/cem2019.pdf>

BRASIIBRASIL. Portaria 1631/GM de 01 de outubro de 2015. Disponível em https://bvsms.saude.gov.br/bvs/saudelegis/gm/2015/prt1631_01_10_2015.html

ESCOREL S, GIOVANELLA L, MENDONÇA MH, MAGALHÃES R, SENNA MCM. Saúde da Família: avaliação da implementação em dez grandes centros urbanos: síntese dos principais resultados. - 2. ed. atual. Brasília: Editora do Ministério da Saúde, 2005; pp 45. Disponível em: http://189.28.128.100/dab/docs/publicacoes/geral/miolo_saude_familia.pdf

ESPERANÇA, Ana Carolina; CAVALCANTE, Ricardo Bezerra; MARCOLINO, Clarice. Estudo da demanda espontânea em uma Unidade de Saúde da Família de uma cidade de médio porte do interior de Minas Gerais, Brasil. Revista Mineira de Enfermagem, [s. I.], v. 10, n. 1, p. 30-36, 2006. Disponível em: <http://www.dx.doi.org/S1415-27622006000100006>

FLOR, Luisa Sorio; CAMPOS, Monica Rodrigues. Prevalência de diabetes mellitus e fatores associados na população adulta brasileira: evidências de um inquérito de base populacional. Revista Brasileira de Epidemiolgia, [s. I.], v. 20, n. 1, p. 16-29, 2017. Disponível em: <https://doi.org/10.1590/19805497201700010002>

GUIBU, lone Aquemi et al. Características principais dos usuários dos serviços de atenção primária à saúde no Brasil. Revista de Saúde Pública, [s. I.], v. 51, n. suppl.2, 2017. Disponível em: <https://doi.org/10.11606/s1518-8787.2017051007070> GUSSO, Gustavo Diniz Ferreira. Diagnóstico de 
demanda em Florianópolis utilizando a Classificação Internacional de Atenção Primária: 2a edição (CIAP2). 2009. [s. I.], 2009.

LANDSBERG, Gustavo de Araújo Porto et al. Análise de demanda em Medicina de Família no Brasil utilizando a Classificação Internacional de Atenção Primária. Ciência \& Saúde Coletiva, [s. I.], v. 17, n. 11, p. 3025-3036, 2012. Disponível em: <https://doi.org/10.1590/S1413-81232012001100019>

MAGNAGO, Renata Faverzani et al. Perfil dos usuários do posto de saúde da família do bairro Humaitá, Tubarão - SC. Arquivos Catarinenses de Medicina, [s. I.], v. 38, n. 2, p. 12-20, 2009. Disponível em:<https://pdfs.semanticscholar.org/f606/fcf236438b986d5ac1cca1d2e09040cabe5c.pdf?_ ga=2.248944901.30925516.1582489534-268487188.1582489534>

MALTA, Deborah Carvalho et al. Prevalência da hipertensão arterial segundo diferentes critérios diagnósticos, Pesquisa Nacional de Saúde. Revista Brasileira de Epidemiologia, [s. I.], v. 21, n. 1, 2018. Disponível em: <https://doi.org/10.1590/1980549720180021.supl.1>

MATUMOTO, Silvia et al. Produção de atendimentos de enfermeiros em unidades da rede básica de saúde. [s. I.], v. 20, n. 4, 2012. Disponível em: <https://doi.org/10.1590/S0104-11692012000400011>

MIRANDA, Pollyane R.; SANTOS, Mário Rogério S.; CHAZAN, Ana Claudia S. Análise de procedimentos ambulatoriais realizados em um centro municipal de saúde do Rio de Janeiro. Revista Hospital Universitário Pedro Ernesto, [s. I.], v. 15, n. 3, p. 235-241, 2016. Disponível em: <https://doi.org/10.12957/rhupe.2016.29449>

NORMAN, Armando Henrique; TESSER, Charles Dalcanale. Acesso ao cuidado na estratégia saúde da família: Equilíbrio entre demanda espontânea e prevenção/promoção da saúde. Saude e Sociedade, [s. I.], v. 24, n. 1, p. 165-179, 2015. Disponível em: <https://doi.org/10.1590/S0104-12902015000100013>

ORGANIZAÇÃO MUNDIAL DE SAÚDE. Classificação Estatística Internacional de Doenças e Problemas Relacionados à Saúde - CID-10. 7. ed. [s.I: s.n.].

ORGANIZAÇÃO MUNDIAL DE SAÚDE. Integração da saúde mental nos cuidados de saúde primários Uma perspectiva global Integração da saúde mental nos cuidados de saúde primários. [s.I: s.n.]. Disponível em:<http://www.who.int/eportuguese/publications/Integracao_saude_mental_cuidados_pri marios.pdf>

NÚCLEO DE TELESSAÚDE SANTA CATARINA. Quais as recomendações para o cálculo da média de consulta/ habitante/ano ou consulta/habitante/hora para os atendimentos médicos, de enfermagem e de odontologia em uma equipe de ESF? Disponível em https://aps.bvs.br/aps/quais-as-recomendacoespara-o-calculo-da-media-de-consultahabitanteano-ou-consultahabitantehora-para-os-atendimentosmedicos-de-enfermagem-e-de-odontologia-em-uma-equipe-de-esf/

PAIM, Jairnilson et al. The Brazilian health system: history, advances, and challenges. The Lancet, [s. I.], v. 377, n. 9779, p. 1778-1797, 2011. Disponível em: <http://dx.doi.org/10.1016/S0140-6736(11)60054-8>

PIMENTEL, Ítalo Rossy Sousa et al. Caracterização da demanda em uma Unidade de Saúde da Família. Revista Brasileira de Medicina de Família e Comunidade, [s. I.], v. 6, n. 20, p. 175-181, 2012. Disponível em: http://www.rbmfc.org.br/index.php/rbmfc/article/view/95\

SAASTAMOINEN, Leena; ENLUND, Hannes; KLAUKKA, Timo. Repeat prescribing in primary care: A prescription study. Pharmacy World and Science, [s. I.], v. 30, n. 5, p. 605-609, 2008. Disponível em: <https://www.ncbi.nlm.nih.gov/pubmed/18509741> SANTOS, Karina de Paula Bastos; 
RIBEIRO, Marco Túlio Aguiar Mourão. Motivos de consulta mais comuns das pessoas atendidas por uma equipe de saúde da família em Fortaleza - CE. Revista Brasileira de Medicina de Família e Comunidade, [s. I.], v. 10, n. 37, p. 1-11, 2015. Disponível em: <http://dx.doi.org/10.5712/rbmfc10(37)831>

STARFIELD, Barbara. Atenção Primária: Equilibrio entre necessidades de saúde, serviços e tecnologia. [s.I: s.n.]. v. 53. Disponível em:<http://www.dominiopublico.gov.br/download/texto/ue000039.pdf>

TOMASI, Elaine et al. Características da utilização de serviços de Atenção Básica à Saúde nas regiões Sul e Nordeste do Brasil: diferenças por modelo de atenção. Ciência \& Saúde Coletiva, [s. I.], p. 4395-4404, 2011.

TORRES, Rinailda de Cascia Santos et al. Principais motivos de consultas médicas em unidades de saúde da família na cidade do Recife, Brasil: um estudo transversal. Sao Paulo Medical Journal, [s. I.], v. 133, n. 4, p. 367-370, 2015. Disponível em: <https://doi.org/10.1590/1516-3180.2014.9490902 > 